\title{
Walter Schaupp, Johann Platzer (Hrsg) (2020) Der verbesserte Mensch. Biotechnische Möglichkeiten zwischen Freiheit und Verantwortung (Bioethik in Wissenschaft und Gesellschaft 11)
}

\author{
Nomos, Baden-Baden, 144 Seiten, 29,00€, ISBN \\ 978-3-8487-6939-1, eBook (Open Access), ISBN \\ 978-3-7489-1056-5, https://doi.org/10.5771/9783748910565
}

\section{Anna Puzio}

Angenommen: 17. März 2021 / Online publiziert: 1. April 2021

(C) Der/die Autor(en) 2021

Durch die technologischen Entwicklungen ist es zu einer Vielzahl an Möglichkeiten gekommen, den Menschen biotechnologisch zu verändern. Zu Zwecken der Therapie oder des Enhancements wird bereits vielfältig auf den Menschen Einfluss genommen. Welche Biotechnologien werden in der Zukunft zur Verfügung stehen? Die verschiedenen wissenschaftlichen Disziplinen wie z.B. die Naturwissenschaften, Ethik und Rechtswissenschaft werden vor neue Herausforderungen gestellt.

Der Sammelband widmet sich diesem aktuellen und relevanten Thema und rïckt „den verbesserten Menschen“ ins Zentrum. In acht kurzen Beiträgen soll die „optimierende Transformation des Menschen" anhand verschiedener Problemfelder exemplarisch beleuchtet werden. Der Sammelband geht auf eine Tagung zurïck, die am 30. September 2019 zum Thema „Natur - Freiheit - Schöpfung. Wie weit sollen wir den Menschen neu erfinden?" in Graz (Universitätszentrum Theologie) stattgefunden hat (S. 5). Eine Besonderheit des Bandes ist seine interdisziplinäre Ausrichtung. Er versammelt Beiträge aus der Rechtswissenschaft, Theologie, Philosophie, Medizin und Ethik, wobei eine bioethische Perspektive alle Aufsätze durchzieht.

Die Beiträge thematisieren den Transhumanismus, die Aneignung von Prothesen und Organen als Teile des Körpers, Geschlechts- und Transidentität, das Genome Engineering und die Optimierung der Psyche. Dabei werden Überlegungen zu Freiheit, Natur und Glück angestellt.

$\mathrm{Zu}$ Beginn nimmt Peter Strasser den Transhumanismus in den Blick. Daran anschließend beleuchtet Reinhold Esterbauer im Spannungsfeld „Leib - Körper - Ma-

\footnotetext{
A. Puzio, Mag. Theol., M. A. ( $ه)$

Westfälische Wilhelms-Universität Münster, Münster, Deutschland

E-Mail: anna.puzio@uni-muenster.de
}

Hochschule für Philosophie München, München, Deutschland 
schine“ (S. 29) phänomenologisch die körperliche Aneignung von Organen, Prothesen und künstlichen Gelenken. Gunda Werner leitet aus Judith Butlers Gendertheorie Impulse für eine „Theologie der Freiheit“ (S. 45) ab. Die beiden folgenden Beiträge widmen sich den gentechnologischen Eingriffen in die Keimbahn. Michael Rosenberger spricht sich aus moraltheologischer und ethischer Perspektive gegen kategorische Verbote von gentechnischen Eingriffen aus. Andreas Reinisch (in einem Interview mit Johann Platzer) gibt Einblicke in die medizinische Praxis und lehnt Keimbahneingriffe grundsätzlich ab. Danach wird Transidentität aus medizinischer (und ethischer) Perspektive in den Vordergrund gerückt (Christian Egarter), woran rechtswissenschaftliche Überlegungen zur Einwilligung von Körperveränderungen (v. a. anhand des Sittenwidrigkeitskorrektivs) anknüpfen (Alois Birklbauer). Der Sammelband schließt mit einem Beitrag des Herausgebers Walter Schaupp zur psychologischen, neurobiologischen und ethischen Glücksforschung, die er in den Kontext des glücksteigernden Enhancements stellt.

Wenn es in der Einleitung heißt, ,es sollen exemplarisch einige Felder angesprochen werden“, in deren Kontext es zu einer Optimierung des Menschen kommt (S. 5), dann trifft dies die Zielsetzung und das Ergebnis des Bandes ganz gut: Es werden weitläufig verschiedene Problembereiche angerissen, aber auch unverbunden aneinandergereiht. Der Band kann einen guten Überblick über relevante Fragestellungen vermitteln, kann sie jedoch nur ansatzweise und oberflächlich beleuchten. Ein konkreter Leitfaden in der Anordnung der Beiträge ist nicht zu erkennen. „Der verbesserte Mensch“, wie es im Titel heißt, wird so weit ausgelegt, dass in einigen Beiträgen die konkreten modernen Biotechnologien aus dem Blick geraten.

Eine Stärke des Sammelbandes ist seine Interdisziplinarität. Die biotechnologischen Veränderungen des Menschen machen einen interdisziplinären Zugang besonders notwendig. Durch mehr Kohärenz des Bandes und eine stärkere Bezugnahme der Beiträge auf ähnliche Themengebiete würden die Erträge des interdisziplinären Ansatzes deutlicher hervortreten.

Die einzelnen Beiträge sind von sehr unterschiedlicher Qualität. So fällt bereits zu Beginn des Bandes auf, dass die Ausführungen zum Transhumanismus von Strasser keinerlei Literatur aufgreifen (bzw. am Ende verallgemeinernd auf zwei eigene Publikationen des Autors als weiterführende Literatur verweisen). Ein Aufsatz über den Transhumanismus sollte die Anforderung erfüllen, sich zumindest auf die Literatur der Transhumanist*innen zu beziehen. Der Beitrag entspricht nicht dem aktuellen Forschungsstand zum Transhumanismus und erscheint somit vielmehr als Meinung des Autors statt als wissenschaftlicher Beitrag. Darüber hinaus werden problematische, diskriminierende Aussagen getroffen wie: „Und so gibt es auch im Fall der Dummheit eine Grenze. Der geistig schwer behinderte, demente Mensch, der die meisten Situationen des alltäglichen Lebens und ihre Folgen nicht richtig deuten und abschätzen kann - mit ihm, sagen wir, stimmt etwas nicht“" (S. 22). Diese werden in einen Zusammenhang mit der Bergpredigt gestellt: „Selig die Armen im Geiste [...]““ (S. 22). Ebenfalls muss das Interview zum Genome Engineering kritisch betrachtet werden, in dem zwar grundlegendes Wissen zu genetischen Eingriffen und zur CRISPR/Cas9-Methode dargelegt wird, eine tiefergehende wissenschaftliche Auseinandersetzung aber ausbleibt. Besonders in den ethischen Aussagen verstrickt sich der Interviewte in Widersprüche, auf die er selbst hinweist (z. B. S. 87). Dies liegt 
aber auch an der Ausrichtung des Interviews, das eigentlich medizinische Aspekte $\mathrm{zu}$ fokussieren bestrebt ist, aber letztendlich die Ethik zum Thema macht.

Dennoch darf nicht aus dem Blick geraten, dass der Band auch ertragreiche, fundierte Beiträge versammelt. Wegweisende und zukunftsoffene Perspektiven für die Lesenden bieten besonders die theologischen, philosophischen und ethischen Beiträge. Sie bieten wertvolle Ausgangspunkte, die sich für die ethische Diskussion biotechnologischer Entwicklungen nutzbar machen lassen.

Der Sammelband zeigt exemplarisch Problemfelder biotechnologischer Verbesserungen des Menschen auf. In der zukünftigen Forschung wird es notwendig sein, den Blick noch gezielter auf die konkreten Biotechnologien zu richten sowie ihre Bedeutung für das menschliche Selbstverständnis, Handeln und körperliche Erleben zu untersuchen. Eine wichtige Aufgabe wird es sein, ethische und rechtliche Normen in den veränderten Kontexten neu auszulegen und zu verändern. Dafür stellen interdisziplinärere Ansätze eine wegweisende Perspektive dar.

Funding Open Access funding enabled and organized by Projekt DEAL.

Open Access Dieser Artikel wird unter der Creative Commons Namensnennung 4.0 International Lizenz veröffentlicht, welche die Nutzung, Vervielfältigung, Bearbeitung, Verbreitung und Wiedergabe in jeglichem Medium und Format erlaubt, sofern Sie den/die ursprünglichen Autor(en) und die Quelle ordnungsgemäß nennen, einen Link zur Creative Commons Lizenz beifügen und angeben, ob Änderungen vorgenommen wurden.

Die in diesem Artikel enthaltenen Bilder und sonstiges Drittmaterial unterliegen ebenfalls der genannten Creative Commons Lizenz, sofern sich aus der Abbildungslegende nichts anderes ergibt. Sofern das betreffende Material nicht unter der genannten Creative Commons Lizenz steht und die betreffende Handlung nicht nach gesetzlichen Vorschriften erlaubt ist, ist für die oben aufgeführten Weiterverwendungen des Materials die Einwilligung des jeweiligen Rechteinhabers einzuholen.

Weitere Details zur Lizenz entnehmen Sie bitte der Lizenzinformation auf http://creativecommons.org/ licenses/by/4.0/deed.de. 